\title{
Effect of Concurrent Partnerships and Sex-Act Rate on Gonorrhea Prevalence
}

\author{
Gavin Welch \\ Department of Epidemiology \\ University of Michigan \\ Ann Arbor, Michigan
}

\author{
Stephen E. Chick \\ Department of Industrial and Operations Engineering \\ University of Michigan \\ Ann Arbor, Michigan
}

\author{
James Koopman \\ Department of Epidemiology \\ University of Michigan \\ Ann Arbor, Michigan
}

The disease gonorrhea (GC) is a major public health problem in the United States, and the dynamics of the spread of GC through populations are complicated and not well understood. Studies have drawn attention to the effect of concurrent sexual partnerships as an influential factor for determining disease prevalence. However, little has been done to date to quantify the combined effects of concurrency and within-partnership sex-act rates on the prevalence of GC. This simulation study examines this issue with a simplified model of GC transmission in closed human populations that include concurrent partnerships. Two models of within-partnership sex-act rate are compared; one is a fixed sex-act rate per partnership, and the other is perhaps more realistic in that the rate depends on the number of concurrent partners. After controlling for total number of sex acts, pseudo-equilibrium prevalence is higher with the fixed sex-act rate than under the concurrency-adjusted rate in all the modeled partnership formation conditions.

Keywords: Epidemic, mixing model, partnership formation, sexually transmitted disease, transmission, concurrency, sex-act rates, gonorrhea

\section{Introduction}

Although effective treatment has been available for the last half-century, the disease gonorrhea (GC) remains a major public health problem in the United States. More than 300,000 cases were reported in the U.S. in 1996, and GC is a major cause of pelvic inflammatory disease, infertility, ectopic pregnancy and chronic pelvic pain [1]. An additional public health concern is that concurrent infection with HIV has been shown to increase the risk of HIV transmission [1]. Along with the human suffering caused by GC, this preventable disease contributes to the $\$ 10$ billion in annual direct and indirect costs resulting from morbidity due to the major sexually transmitted diseases (STDs), excluding HIV/ AIDS [2].

Neisseria gonorrhea, the bacterial pathogen which causes GC, is perhaps one of the best understood human pathogens from a microbiologic point of view. However, the GC transmission system which consists of the contact patterns of the human hosts, or which members of the population engage in sexual activity with each other, and host immune system-pathogen interactions, is complex and not well understood.

One part of the transmission system that has not been elucidated to date is the effect of within-sexualpartnership sex-act rates on the prevalence of disease in a population that is not strictly monogamous. Over the length of a sexual partnership, one or both partners can have multiple partners at a point in time, or concurrent partners, from whom they can become 
infected or transmit infection to. The effect of concurrent partnerships on disease prevalence has been demonstrated in models by several authors $[3,4,5,6,7]$.

These models have, however, assumed a constant rate of transmission per partnership in continuous compartmental models (CCM) $[3,4,5]$, or constant risk of transmission per day between infected and non-infected partners in the stochastic models of Kretzschmar and Morris [6, 7]. Evidence that concurrent partners can affect the sex-act rate within a partnership has been presented by Blower and Boe [8].

The effects of concurrency and sex-act rates on disease prevalence cannot be evaluated without considering the contact pattern of the human host population. The importance of contact pattern for GC was presented by Yorke, Hethcote and Nold [9] and Hethcote and Yorke [10], who used CCMs to demonstrate the need for a small, highly sexually active subgroup in the host population for GC to remain endemic. With the advent of the AIDS epidemic, many CCMs (for example, [11]) were formulated that showed the rate of new partner acquisition, as well as non-random contact patterns, were important factors in the spread of HIV and other STDs. While these models provided great insight into STD epidemiology, they have, for the most part, modeled disease incidence and partnership formation as continuous flows, which makes it difficult or impossible to analyze the effects of patterns of sex acts and concurrency within partnerships on disease prevalence.

To evaluate the effect of sex-act rates within partnerships on disease prevalence, this paper builds on the classical mixing model of Hethcote et al. by analyzing simulations of a stochastic process model that includes non-random partnership formation, partnerships that have a defined length, concurrent partnerships and a defined within-partnership sex-act rate. Disease prevalence under two sex-act rates, one a function of concurrent partners and one independent of concurrency, is analyzed.

\section{Methods}

The methods section contains three main sub-sections. The first presents a conceptual description of the model that was used to control the formation of sexual partnerships among the simulated humans. The second contains the definitions of the two different models that were used to define sex-act rates within partnerships, and the third describes the experiment that compared disease pseudo-equilibrium prevalence between the two sex-act rate models.

\subsection{Partnership Formation Model}

The pattern of sexual partnership formation within the simulated population, or mixing pattern, was central to this investigation. As discussed in the introduction, mixing patterns can greatly affect disease prevalence. For this study it was necessary to control who formed partnerships with whom, as well as the characteristics of the resulting partnerships, so that the effects of the sex-act rate assumptions within partnerships could be isolated and evaluated independently of the effects of the mixing pattern. The model of the partnership formation process used in this project allows explicit definition of the types of partnerships that can form, the rate at which each type of partnership forms, and the quantity of concurrent partnerships in the population. The model is described here in a general form and could also be applied to other simulation studies involving sexual partnership formation or other forms of non-random contact (needle sharing among intravenous drug users, for instance). The specific parameters used in this study are given in Section 2.3.

The model assumes that a finite number of different types of partnerships can be formed in the simulated population. The types of partnerships and their characteristic parameters are defined by the investigator. Characteristics describing a type of partnership could include, but are in no way limited to, length of partnership, type of sexual activity desired by the partners, ages of the partners, etc. Examples of partnership types could be: long-term partnerships between humans 20 to 30 years old, or, one-sex-act partnerships between commercial sex workers and their clients. By model definition, sex acts can only occur within the context of a sexual partnership.

In addition to humans who form partnerships, the partnership formation model has two major components: "mixing bins," which generate partnerships, and "tokens," which are assigned to humans and control the number of partnerships any human can be in at any point in time. There is one mixing bin in the model for each type of partnership. Each mixing bin generates new partnerships of its defined partnership type at the rate specified by the investigator as long as there are tokens in the unpartnered state representing humans eligible to form partnerships in the mixing bin. Each human in the model has one token for each partnership it can be in concurrently. For example, a human with one token will always be either unpartnered or have one partner. A human with three tokens can have zero, one, two or three partners at any point in time. Each token can be in one of two states: partnered or unpartnered. A token in the unpartnered state represents the availability of its owner to form a sexual partnership; a token in the partnered state indicates that the human is involved in a partnership.

A partnership can form between two humans if:

1. Each of them has a token in the unpartnered state,

2. The model rules governing partnership formation allow them to form a partnership (for example, if only heterosexual couples are allowed, two males or two females could not form a partnership), and

3. They are not already in a partnership with each other. 
Time 1

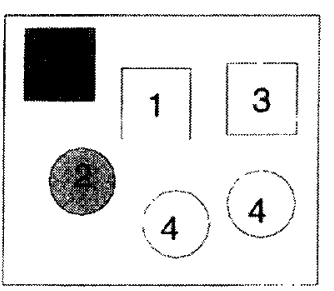

Partnerships:

$1-2$

Potential Partnerships:

$1-4$

$3-4$
Time 2

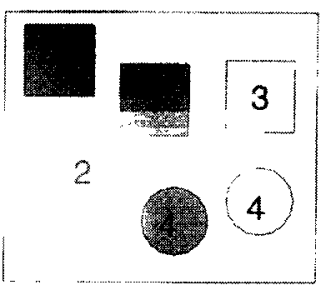

Partnerships:

$1-2$

$1-4$

Potential Partnerships:
Time 2.5

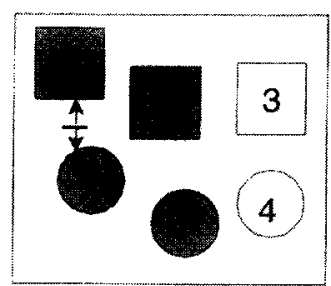

3-4

Time 3

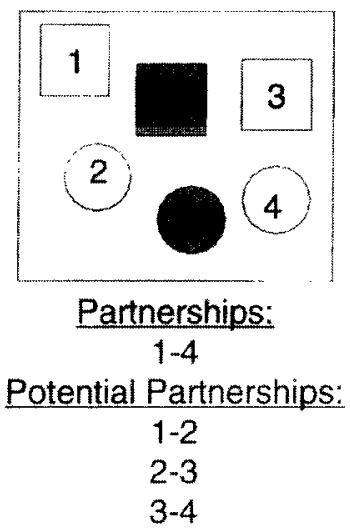

Legend:

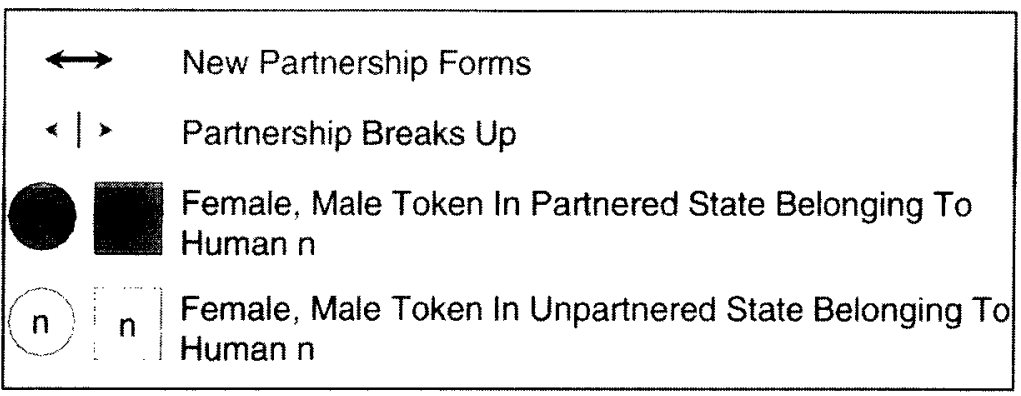

Figure 1. Five snapshots of a mixing bin taken at consecutive times show partnership formation and breakup. Detailed description is provided in the Partnership Formation Model section of the text.

When two humans form a partnership, the token of each human who was in the unpartnered state is set to the partnered state. When the partnership ends, the two tokens are returned immediately to the unpartnered state and the humans who were in the partnership are again available to join new partnerships. To control the type(s) of partnerships(s) each human can enter into, tokens are assigned to one of the mixing bins in the model. For instance, a human with three tokens might have one in a mixing bin that generated long-term partnerships, and his or her remaining two tokens in a bin that outputs short-term "one-night stands." Two humans must each have at least one token in the same mixing bin in order to be able to enter into a partnership with each other.

To illustrate how a mixing bin works, Figure 1 shows a diagram of a heterosexual partnership producing mixing bin containing six tokens belonging to four different humans, two female and two male. Snapshots of the mixing bin at five consecutive times are presented: at each pictured time point, the existing partnerships and all possible partnerships are listed. At time 1, the only existing partnership is between humans 1 and 2. Human 2 has no unpartnered tokens in the mixing bin, but humans 1,3 and 4 can still enter into partnerships of the type generated by the mixing bin. A partnership between human 1 and human 4 is formed at time 1.5, and at time 2 the only humans with available tokens in the mixing bin are human 3 and human 4, and so the only partnership that can form is between humans 3 and 4 . At Time 2.5 the partnership between human 1 and human 2 breaks up; after that (Time 3 is shown), all the humans with tokens in the mixing bin have unpartnered tokens and can form new partnerships. Humans 1 and 4 are already partnered and so cannot form another partnership with each other, however.

Each mixing bin randomly produces new partnerships with an exponentially distributed rate defined by the investigator as long as it is possible for new partnerships to form (i.e., there are tokens belonging to male and female humans who are not already partnered in the mixing bin). The mean length of partnerships formed by a mixing bin is the inverse of an investigator-defined breakup rate parameter. Partnership lengths are random, independent and exponentially distributed. Defining parameters for average partnership length provides useful information about the number of each type of partnership present because by specifying the rate at which a type of partnership forms and its average length, the expected number of partnerships of any type in existence when the model 
is at equilibrium is implicitly defined. The use of exponential distributions allows for certain special cases of the mixing model (one mixing bin model) to be analyzed in closed form as a birth-death process. This feature assisted with model validation.

\subsection{Sex-Act Rates}

The two sex-act rates compared in this investigation were termed a "fixed" sex-act rate and a "concurrencyadjusted" sex-act rate. For the fixed sex-act rate, each partnership started with a sex act and after every sex act, the time to the next sex act in the partnership was taken from an exponential distribution with an arbitrarily chosen mean rate of $1 / 0.43$, or about three times a week. Simulated sex acts were generated at the fixed rate until the partnership ends.

Under the concurrency-adjusted sex-act rate, each new partnership also began with a sex act. Additional sex acts within the partnership were generated at a rate inversely proportional to the total number of concurrent partners of each partner. This rate was recalculated after each sex act in the partnership. For example, if $A$ and $B$ are partnered, and $A$ has a total of $N_{A}$ partners, and $B$ has a total of $N_{B}$ partners, then the concurrency-adjusted sex-act rate is proportional to $1 /\left(N_{A}\right.$ $\left.+N_{B}\right)$. A constant of proportionality was chosen so that the total number of sex acts in the entire population per unit time would be the same for both the fixed and concurrency-adjusted sex-act rate simulations. Without the adjustment parameter, the number of sex acts in a simulation running with the fixed sexact rate would have been higher than the number occurring in a simulation that was identical, but run with the concurrency-adjusted sex-act rate. This disparity in number of sex acts, or potential opportunities for disease transmission, could have explained any observed differences in disease pseudo-equilibrium prevalence, and so it had to be controlled for.

\subsection{Experiment}

In order to determine if disease pseudo-equilibrium prevalence differed between the fixed and concurrencyadjusted sex-act rate assumptions, a complete block experimental design was used to compare pseudoequilibrium prevalence under both rates at two levels of three factors that have been shown to affect GC prevalence. The three two-level factors included in the experiment were:

1. Amount of concurrency defined by the percentage of the high and low sexual activity population subgroups capable of having concurrent partners and the average number of concurrent partners among those capable of concurrency.

2. Amount of mixing between the high and low sexual activity groups defined by the percentage of partnerships within each group that were formed in mixing bins that allowed high and low activity group members to form partnerships.

3. Amount of sexual activity defined by the new partnership formation rates of the mixing bins.

Parameters describing the levels of the factors amount of concurrency and between-group mixing are displayed in Table 1. Four mixing bins regulated partnership formation rates (factor 3 ) and the types of partnerships that could be formed; the bins are described in Table 2. Mixing bin partnership formation rates were calculated using the structured mixing formulation of Jacquez et al. [12] so that the expected number of new partnerships per year in the high activity group was 30 and 50 at the low and high partnership formation rate settings, respectively, and 1.1 and 1.5 , respectively, for the low activity group.

The number of tokens assigned to a population subgroup was one token for each member of the group, plus one token for each possible concurrent partnership that concurrency-capable humans in the subgroup could be in. All humans in a subgroup were randomly

Table 1. Levels of factors affecting the high and low activity population subgroups. Percent partnerships in bin $\{1,2,3,4\}$ is the expected percentage of each activity group's partnerships that form in each of the four mixing bins. Proportion capable of concurrency is the proportion of humans in a population subgroup who can have concurrent partnerships; average concurrency tokens is the average number of concurrent partnerships in excess of one assigned to those humans.

\begin{tabular}{|c|c|c|c|c|c|}
\hline Factor & Level & \multicolumn{2}{|c|}{ High Activity Group } & \multicolumn{2}{|c|}{ Low Activity Group } \\
\hline \multirow[t]{2}{*}{$\begin{array}{l}\text { Between- } \\
\text { Group } \\
\text { Mixing }\end{array}$} & $\begin{array}{l}\text { Low } \\
\text { High }\end{array}$ & \multicolumn{2}{|c|}{$\begin{array}{c}\text { Percent Partnerships in Bin }\{1,2,3,4\} \\
\{0.0,2.5,2.5,95.0 \\
\{0.0,10.0,10.0,80.0\}\end{array}$} & \multicolumn{2}{|c|}{$\begin{array}{c}\text { Percent Partnerships in Bin }\{1,2,3,4\} \\
\{95.0,2.5,2.5,0.0 \\
\{80.0,10.0,10.0,0.0\}\end{array}$} \\
\hline & & $\begin{array}{l}\text { Proportion } \\
\text { Capable of } \\
\text { Concurrency }\end{array}$ & $\begin{array}{l}\text { Average } \\
\text { Concurrency } \\
\text { Tokens }\end{array}$ & $\begin{array}{l}\text { Proportion } \\
\text { Capable of } \\
\text { Concurrency }\end{array}$ & $\begin{array}{l}\text { Average } \\
\text { Concurrency } \\
\text { Tokens }\end{array}$ \\
\hline \multirow[t]{2}{*}{ Concurrency } & Low & 0.8 & 4 & 0.1 & 1 \\
\hline & High & 1.0 & 5 & 0.2 & 2 \\
\hline
\end{tabular}


Table 2. Expected partnerships formed per day in each mixing bin. Activity group indicates whether the "high" activity group, "low" activity group, or both have tokens in the bin.

Expected partnership length is the inverse of the break up rate for the mixing-bin.

\begin{tabular}{|c|c|c|c|c|c|c|c|}
\hline \multirow{3}{*}{$\begin{array}{c}\text { Mixing } \\
\text { Bin }\end{array}$} & \multirow[b]{3}{*}{$\begin{array}{c}\text { Activity } \\
\text { Group (High, } \\
\text { Low, Both) } \\
\end{array}$} & \multirow[b]{3}{*}{$\begin{array}{c}\text { Expected } \\
\text { Partnership } \\
\text { Length (days) } \\
\end{array}$} & \multicolumn{5}{|c|}{ Mixing Bin Output Partnerships/Day } \\
\hline & & & \multirow{2}{*}{$\begin{array}{l}\text { Factor: } \\
\text { Partnership Formation Rate: } \\
\text { Between-Group Partnerships: }\end{array}$} & \multicolumn{4}{|c|}{ Level: } \\
\hline & & & & $\begin{array}{l}\text { Low } \\
\text { Low }\end{array}$ & $\begin{array}{l}\text { Low } \\
\text { High }\end{array}$ & $\begin{array}{l}\text { High } \\
\text { Low }\end{array}$ & $\begin{array}{l}\text { High } \\
\text { High }\end{array}$ \\
\hline 1 & Low & 200 & & 1.35 & 1.14 & 1.85 & 1.56 \\
\hline 2 & Both & 60 & & 0.09 & 0.35 & 0.13 & 0.56 \\
\hline 3 & Both & 3 & & 0.09 & 0.35 & 0.13 & 0.56 \\
\hline 4 & High & 3 & & 1.95 & 1.64 & 3.25 & 2.74 \\
\hline
\end{tabular}

assigned one token from all the tokens available to the subgroup. Each human in the group capable of concurrency was randomly assigned one more token so that concurrency-capable humans had at least two tokens. Remaining concurrency tokens (if any) were then randomly assigned to the concurrency-capable humans in the group. Tokens were assigned to mixing bins based on the percentage of each subgroups' partnerships that were expected to form in each mixing bin. For example, in a simulation where the between-group mixing factor was set to low, $95 \%$ of the high activity group's tokens would be assigned to bin 4, which produced short partnerships at a high rate. The number of male and female tokens in each mixing bin was the same for this investigation.

The simulated population consisted of 500 male and 500 female humans who could form heterosexual partnerships and engage in sex acts with their partners over the course of the simulation. Jacquez and Simon [13] have shown that for stochastic models of closed population susceptible-immune-susceptible (SIS) epidemics, population sizes of 100 or more produce a pseudo-equilibrium prevalence that is close to that observed in a comparable deterministic model. Jacquez and Simon also note that the true equilibrium prevalence for a stochastic closed SIS model is 0, although it may take an extremely long time to reach. Because of this finding, we refer to pseudo-equilibrium prevalence when discussing the results of this experiment. The population size of 1,000 is also consistent with other recent GC simulation models [14]. For this investigation, the population was closed with no births, deaths or migration. There were two population subgroups in this experiment; $5 \%$ of each sex was in the "high" sexual activity subgroup that formed more partnerships and had a higher predilection for having concurrent partners; the remainder of the population was in the "low" activity subgroup. Sexual activity was the only subsetting characteristic of the population. Yorke, Hethcote and Nold [9] estimated the size of the core, or high activity, group, to be about $2.5 \%$ of the at risk population. The $5.0 \%$ parameter used in this investigation was to insure that the simulated epidemics did not die out.

The simulated GC pathogen was transmissible with defined probability at each sex act between an infected and uninfected human. There was one type of sex act in the simulation, analogous to vaginal intercourse. The transmission probability per sex act from infected female to uninfected male during vaginal intercourse has been estimated at 0.19 and 0.53 [15]. The risk of transmission from infected male to uninfected female has not been measured, but is generally thought to be much higher than from female to male. For this investigation, the probability of transmission from female to male was 0.30 per sex act, and for male to female, 0.70 per sex act. The transmission probabilities were adjusted upward from the literature values to insure that the simulated epidemics did not die out.

For this investigation, GC was modeled as a susceptible-immune-susceptible (SIS) disease. Immediately upon infection, a newly infected human became infectious and remained infectious until his or her infection ended. Infection did not result in any immunity, and an infected human was susceptible again immediately after an exponentially distributed infectious period. Mean recovery rates for females was $1 / 45$ per day and for males, $1 / 30$ per day. Although not biologically plausible, the exponential infection times were used to maintain consistency with CCM. Yorke, Hethcote and Nold [9] cite previous modelers as having used mean infectious period lengths of 10,55 and 100 days for infected males, both sexes, and females, respectively. Recovery rates in this simulation were based on the 55-day estimate and adjusted downward to implicitly include treatment. Females had a slower recovery rate than males to take into account their higher likelihood of having an asymptomatic and undetected infection. Contact tracing of the partners of infected humans was not included in the simulation.

Thirty simulations at each of the $2^{4}$ combinations of the levels of factors were run. Pairs of fixed and 
concurrency-adjusted runs used the same population at each combination of the three factors. One hundred humans were randomly chosen to become infected in all experimental runs when the partnership process reached approximately equilibrium (simulation day 1,800 , or about five years). The simulation outcome was chosen to be the percentage of the population that was infected when the infection process reaches pseudoequilibrium (simulation day 3,480 , or about 9.5 years). This time was selected by visual inspection of infection levels in the population as a function of time.

Differences in pseudo-equilibrium prevalence at different combinations of levels of the experimental factors were tested for using an analysis of variance. The model included main effects for type of sex-act rate (fixed versus concurrency-adjusted) and the three factors, as well as three sex-act rates by factor interaction terms. The analysis of variance was done with SAS Version 6.12 PROC ANOVA (SAS Institute, Cary, NC).

The simulation program was written in Kernighan and Ritchie C, and compiled with the GNU C compiler (Free Software Foundation, Boston, MA). Uniform random numbers for the simulation were generated with the "ran2" subroutine from Numerical Recipes in C [16].

\section{Results}

The means of the measured prevalences for all settings at fixed and concurrency-adjusted levels of the sex-act rate parameter are displayed in Table 3. Mean prevalence was consistently highest at the fixed sex-act level, even when the average number of sex acts was higher in the concurrency-adjusted level (sex-act data not shown). The effect was much more pronounced at high concurrency settings, however. High concurrency settings also consistently had higher prevalences than their low concurrency setting analogues. A similar effect is observable for high compared to low part- nership formation rates. Mean prevalences at all settings comparing fixed and concurrency-adjusted results are shown graphically in Figure 2.

The analysis of variance model used to evaluate factor effects on GC prevalence had a significant $(p<0: 05)$ overall F-test for the existence of at least one factor effect. Model effects that differed significantly from 0 were: sex-act rate, concurrency, partnership formation rate and the sex-act rate by concurrency interaction. The main effect for between-group mixing did not differ significantly from 0 .

\section{Discussion}

In this investigation, pseudo-equilibrium prevalence of GC under two different within-partnership sex act scheduling methods, fixed rate and concurrency adjusted, was compared after controlling for the effect of number of sex acts in the population as a whole. In the simulated closed population, sex-act rate method did affect disease prevalence. The size of the effect was smaller than the effects seen for changing the amount of concurrency or partnership formation rate, but the results do suggest that assuming a fixed sex-act rate within all partnerships, or fixed risk of transmission per day in all partnerships regardless of number of concurrent partners, could cause an overestimation of prevalence in models of GC or other diseases that can be described by an SIS model.

Many investigators have evaluated the effect of concurrency on disease transmission dynamics. Dietz and Hadeler [4] modeled an SIS infection in a randomly mixing population with a CCM that included concurrent partners and partnership length; they concluded that the transmission dynamics of the infection in their model were dependent on contact rate within the partnership and length of the partnership. Watts and May [3] presented a CCM of HIV in a randomly

Table 3. Mean point prevalence (percentage of population infected) of 30 repetitions at each experimental setting under fixed and concurrency-adjusted sex-act rate in the simulated population of 1,000 humans.

\begin{tabular}{|c|c|c|c|c|c|}
\cline { 2 - 6 } \multicolumn{1}{c|}{} & \multicolumn{3}{c|}{ Factor Levels } & $\begin{array}{c}\text { Mean Percent of Population Infected } \\
\text { When Sex-Act Rate Was: }\end{array}$ \\
\hline Setting & $\begin{array}{c}\text { Partnership } \\
\text { Formation Rate }\end{array}$ & $\begin{array}{c}\text { Between-Group } \\
\text { Partnerships }\end{array}$ & Concurrency & Fixed & Adjusted \\
\hline 1 & Low & Low & Low & 2.80 & 2.57 \\
\hline 2 & Low & Low & High & 11.05 & 9.00 \\
\hline 3 & Low & High & Low & 3.34 & 2.84 \\
\hline 4 & Low & High & High & 10.34 & 8.81 \\
\hline 5 & High & Low & Low & 4.52 & 4.14 \\
\hline 6 & High & Low & High & 26.39 & 24.63 \\
\hline 7 & High & High & Low & 6.59 & 6.01 \\
\hline 8 & High & High & High & 24.60 & 22.31 \\
\hline
\end{tabular}




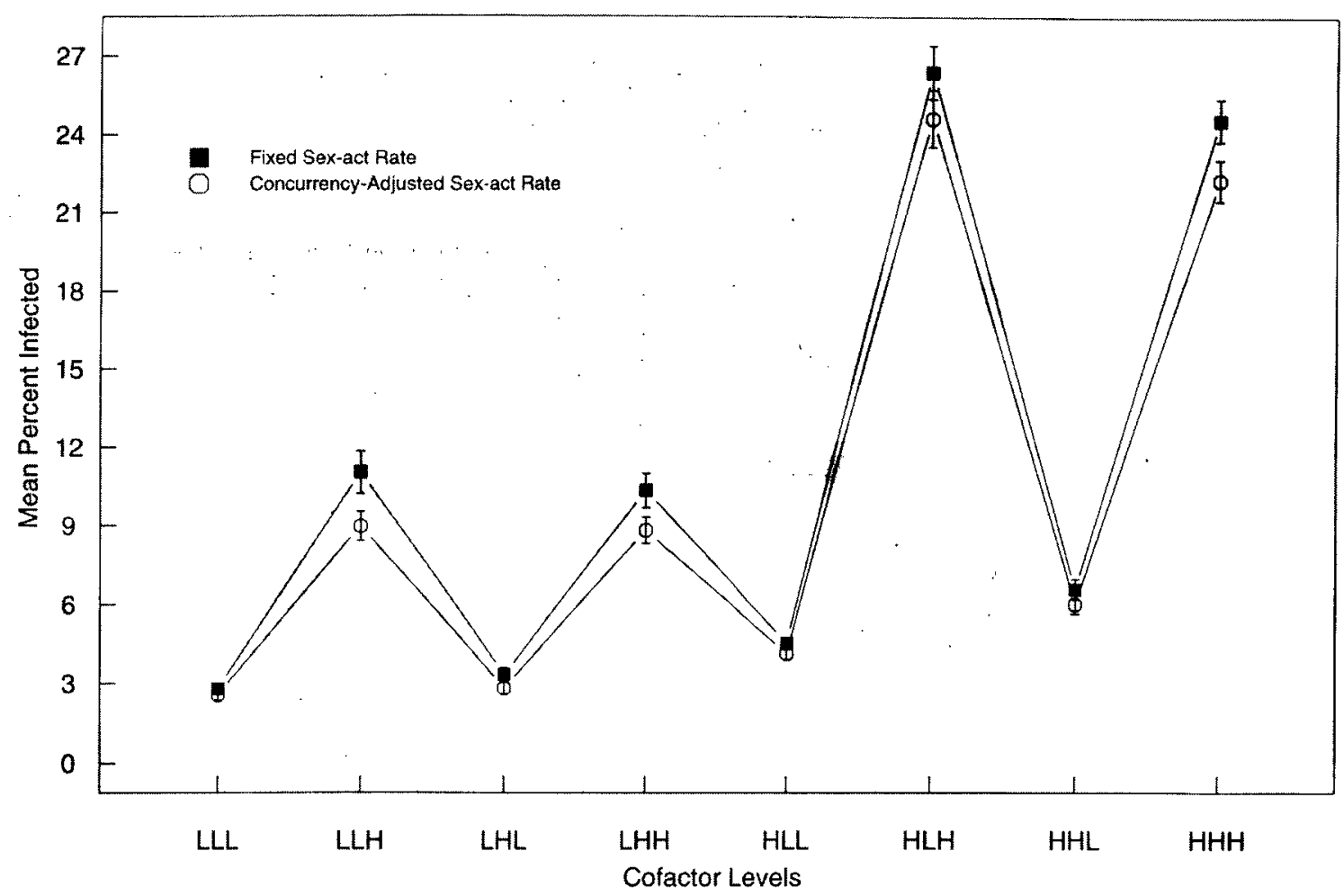

Figure 2. Mean pseudo-equilibrium prevalence (percentage of the population infected) under fixed and concurrency-adjusted sex-act rates at the eight experimental settings defined in Table 3 . Error bars denote upper and lower $95 \%$ confidence intervals. Factor levels on the X-axis refer to the low or high settings of partnership formation rate, between-group partnership formation and concurrency as described in Tables 1 and 2.

mixing population that included concurrent partnerships and length of partnership: their results focus on the effect of concurrency on the rate of spread of the epidemic. Altmann [5] has published a model which allows for multiple concurrent partnerships and assumes that contacts occur at a constant rate within partnerships. The stochastic models of Kretzschmar and Morris $[6,7]$ have demonstrated the strong effect of concurrency on the prevalence of GC like SIS diseases. The results of this study are consistent with the findings of these previously published results. The lack of effect from between-group mixing is also consistent with the results of Stigum et al. [17], whose CCM did not show a large effect on disease prevalence until their high and low activity groups in their model were mixing nearly randomly.

The results of the simulation showed that in simple cases, how sexual partnerships plan the time until their next sex act can affect disease prevalence. Asking how generalizable the findings are to real human populations is an important step for any epidemic simulation. This model compared the effects of two very simple methods of determining when sex acts occur within a sexual partnership, one of which included concurrent partners in the decision. Important assumptions were made with respect to:

1. Sex-act rate is homogeneous within the population, 2. The per-sex-act transmission probabilities used, and
3. Infection with GC does not result in a protective immune response.

It is extremely unlikely that every sexual partnership in a population uses the same formula to decide when their next sex act will occur. But this assumption does not detract from the finding that if concurrency affects that decision, it is possible that it can affect disease prevalence. Blower and Boe [8] have hypothesized the existence of a "sex budget" where the members of a population have approximately the same number of sex acts, but they may differ in the number of people they have sex with over a given time period. Rather than attempt to solve the potentially very complex problem of ensuring that all the members of the population "spend" their sex budgets in the allotted time, the model used here expands the definition of partnership from the classic SIS model using two different assumptions about the sex-act rate within partnerships. To address assumption two, additional simulation experiments were performed to evaluate the sensitivity of the observed results to changing transmission probabilities (data not shown). The differences in pseudo-equilibrium prevalence were more pronounced as lower transmission probabilities were used, until the transmission probabilities approached 0 .

It has long been believed that GC infection does not result in a protective immune response due to the incidence of repeat infection and multiple methods of host defense evasion that $N$. gonorrhea is capable of 
(reviewed in [18]). Some epidemiologic studies have suggested otherwise [19] and substantial evidence of a host immune response against GC infection has been documented [20]. To our knowledge, immune response has not been integrated into models of GC transmission to date. We hope to expand our model by adding multiple immunologically distinct pathogens that will induce a host immune response dependent on the host's previous infection and exposure status.

\section{References}

[1] Division of STD Prevention. Sexually Transmitted Disease Surveillance. U.S. Department of Health and Human Services, Public Health Service, Centers for Disease Control and Prevention, Atlanta, September 1997.

[2] Institute of Medicine (U.S.), Committee on Prevention and Control of Sexually Transmitted Diseases. The Hidden Epidemic: Confronting Sexually Transmitted Diseases, T. R. Eng and W. T. Butler, editors. Committee on Prevention and Control of Sexually Transmitted Diseases, Institute of Medicine, Division of Health Promotion and Disease Prevention, 1997.

[3] Watts, C. H. and May, R. M. "The Influence of Concurrent Partnerships on the Dynamics of HIV/AIDS." Mathematical Biosciences, Vol. 108, No. 1, pp 89-104, February 1992.

[4] Dietz, K. and Hadeler, K. P. "Epidemiological Models for Sexually Transmitted Diseases." Journal of Mathematical Biology, Vol. 26, No. 1, pp 1-25, 1988.

[5] Altmann, M. "Susceptible-Infected-Removed Epidemic Models with Dynamic Partnerships." Journal of Mathematical Biology, Vol. 33, No. 6, pp 661-75, 1995.

[6] Kretzschmar, M., Van Duynhoven, Y. T, and Severijnen, A. J. "Modeling Prevention Strategies for Gonorrhea and Chlamydia Using Stochastic Network Simulations." American Journal of Epidemiology, Vol. 144, No. 3, pp 306-317, August 1, 1996.

[7] Morris, M. and Kretzschmar, M. "Concurrent Partnerships and Transmission Dynamics in Networks." Social Networks 1995, pp 1-20, 1995.

[8] Blower, S. M. and Boe, C. "Sex Acts, Sex Partners, and Sex Budgets: Implications for Risk Factor Analysis and Estimation of HIV Transmission Probabilities." Journal of Acquired Immune Deficiency Syndromes, Vol. 6, No. 12, pp 1347-1352, December 1993.

[9] Yorke, J. A., Hethcote, H. W. and Nold, A. "Dynamics and Control of the Transmission of Gonorrhoea." Sexually Transmitted Diseases 1978, No. 5. pp 51-56, 1978.

[10] Hethcote, H. W. and Yorke, J. A. Lecture Notes in Biomathematics 56 Gonorrhea Transmission Dynamics and Control, SpringerVerlag, New York, 1984.

[11] Anderson, R. M. “The Epidemiology of HIV Infection: Variable Incubation Plus Infectious Periods and Heterogeneity in Sexual Activity." Journal of the Royal Statististical Society, A 1988, 151, Part 1, pp 66-93, 1988.

[12] Jacquez, J. A., Simon, S. and Koopman, J. S. "Structured Mixing: Heterogeneous Mixing by the Definition of Activity Groups." Lecture Notes in Biomathematics, Castillo-Chavez, editor, pp 316-348.

[13] Jacquez, J. A. and Simon, C. P. “The Stochastic SI Model with Recruitment and Deaths. I. Comparison with the Closed SIS Model." Mathematical Biosciences. Vol. 117, Nos. 1-2, pp 77-125, Sept-Oct 1993.

[14] Ghani, A. C., Swinton, J. and Garnett, G. P. "The Role of Sexual Partnership Networks in the Epidemiology of Gonorrhea." Sexually Transmitted Diseases, Vol. 24, No. 1, pp 45-56, January 1997.

[15] Hooper, R. R., Reynolds, G. H., Jones, O. G., et al. "Cohort Study of Venereal Disease. I: The Risk of Gonorrhea Transmission from Infected Women to Men." American Journal of Epidemiology, Vol. 108, No. 2, pp 136-44, August 1978.

[16] Press, W. H., Teukolsky, S. A., Vetterling, W. T. and Flannery, B. P. Numerical Recipes in C: The Art of Scientific Computing, Cambridge University Press, New York, 1988.
[17] Stigum, H., Falck, W, and Magnus, P. "The Core Group Revisited: The Effect of Partner Mixing and Migration on the Spread of Gonorrhea, Chlamydia, and HIV." Mathematical Biosciences, Vol. 120, No. 1, pp 1-23, March 1994.

[18] Sparling, P. F., Tsai, J. and Cornelissen, C. N. "Gonococci are Survivors." Scandinavian Journal of Infectious DiseasesSupplementum, Vol. 69, pp 125-136, 1990.

[19] Plummer, F. A., Simonsen, J. N., Chubb, H., Slaney, L., Kimata, J., Bosire, M., Ndinya-Achola, J. O. and Ngugi, E. N. "Epidemiologic Evidence for the Development of SerovarSpecific Immunity after Gonococcal Infection." Journal of Clinical Investigation, Vol. 83, No. 5, pp 1472-1476, May 1989.

[20] Brooks, G. F. and Lammel, C. J. "Humoral Immune Response to Gonococcal Infections." Clinical Microbiology Reviews, 2 Suppl: S5-10, April 1989.

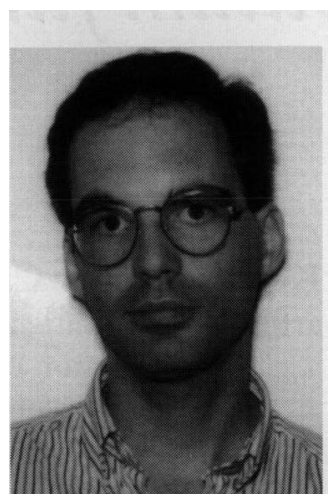

Gavin Welch is a Research Associate in the Department of Epidemiology at the University of Michigan School of Public Health. He has a BS in Microbiology from the University of Maine and an MSPH in Epidemiology from the University of South Carolina School of Public Health. Welch is currently completing the requirements for a $\mathrm{PhD}$ in Epidemiology at the University of Michigan. His research interests are in the analysis of infectious disease transmission systems and the effects of host immunity on pathogen distribution.

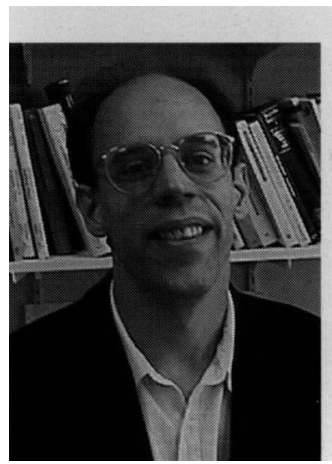

Stephen E. Chick is an Assistant Professor of Industrial and Operations Engineering at the University of Michigan. He received his PhD in 1995 from the Department of Industrial Engineering and Operations Research at the University of California at Berkeley. In addition to stochastic simulation, Chick's research interests include Bayesian statistics, decision analysis, computational methods in statistics, and applications in health care, particularly epidemiology. His work experience includes material handling system design for the automotive industry using simulation analysis.

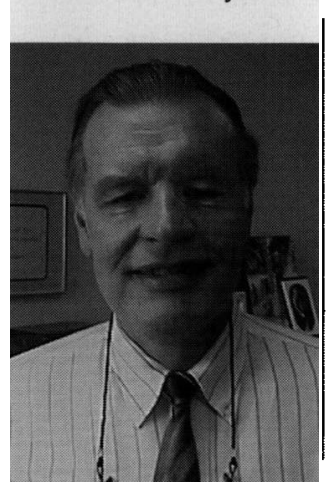

James Koopman is Professor of Epidemiology at the University of Michigan, where he also received his medical degree. He now dedicates himself to developing a science of transmission system analysis. In this endeavor, he helps organize public health department efforts to control infectious diseases and designs and analyzes transmission system models. Koopman views the science of transmission system analysis as pursuing divergent paths within the disciplines of applied mathematics and field epidemiology. He now works with mathematicians, operations engineers and computer scientists to help give common directions to this science. 